\title{
Perpendicular Magnetic Anisotropy and Magneto-Optical Kerr Effect in Giant Magnetoresistive Co-Ag Granular Thin Films
}

\author{
Y.J. Chen, T. Suzuki, G. Phillips, Y. Itoh, W.Y. Cheung", and H. Sang** \\ Information Storage Materials Lab., Toyota Tech. Inst., 2-12-I Hisakata, Tempaku-ku, Nagoya 468-8511, Japan \\ * Department of Electronic Engineering. The Chinese University of Hong Kong \\ **Institute for Solid State Physics, Nanjing University, Nanjing 210093, China
}

The magneto-optical Kerr effect in $\mathrm{Co}_{22} \mathrm{Ag}_{78}$ granular thin films which exhibit a perpendicular magnetic aniostropy is studied using a Kerr spectroscope at photon energy of $1.4-6.8 \mathrm{eV}$. The observed shift of both the positive peak at $5-6 \mathrm{eV}$ of Kerr rotation angle and the negative peak at $4-5 \mathrm{eV}$ of the Kerr ellipticity toward lower energies with increasing annealing temperature suggests the change in electronic structures of fine Co particles / the change of the shape of the particles compared with that of previously reported of FCC Co.

Key words: magnetic anisotropy, magneto-optical spectroscopy, stripe domain, magnetic granular thin film, giant magnetoressistance (GMR), magnetic force microscopy (MFM)

\section{Introduction}

Magneto-optical spectroscopy is widely used for investigating the magnetic properties and electronic band structures of magnetic materials. Recently, the study of magnetic granular metallic films showed quite different magneto-optical spectra from those of pure $\mathrm{Co}$ or $\mathrm{Fe}^{1,2)}$ However, the magneto-optical measurements were performed only in a range of $1.5-5.5 \mathrm{eV}$ of the incident light. In this work, we present the results of the magnetooptical Kerr spectroscopic measurements in a short wavelength region (blue to ultra-violet) in $\mathrm{Co}_{22} A \mathrm{~g}_{78}$ granular thin films. These samples were optimized for a giant magnetoressistance (GMR) ratio. ${ }^{3)-6)}$ Magnetic force microscopy (MFM) observations of these samples showed magnetic stripe domains of much larger size (typically of $100 \mathrm{~nm}$ wide) than that of Co particles $(\sim 10 \mathrm{~nm}$ in diameter), indicating the existence of a perpendicular magnetic anisotropy. ${ }^{7)}$ To understand the mechanism of the origin of this perpendicular anisotropy, a systematic study has been carried out in $\mathrm{Co}_{22} \mathrm{Ag}_{78}$ granular thin films.

\section{Experimental}

$\mathrm{Co}_{22} \mathrm{Ag}_{78}$ films with the thickness of $500-800 \mathrm{~nm}$ were prepared on glass substrate by ion-beam cosputtering at substrate-deposition temperature of $300 \mathrm{~K}$, followed by a postannealing in vacuum at different annealing temperatures $T_{a}$ up to $700 \mathrm{~K}$. Details of the fabrication process and $\mathrm{x}$-ray diffraction (XRD), transmission electron microscopy (TEM), ferromagnetic resonance (FMR), and magnetoresistance (MR) measurements have been published elsewhere. ${ }^{3)-6}$ The magnetic domains were imaged under ambient conditions by a Nanoscope III scanning probe microscope (SPM) and also a Dimension 3000 SPM (Digital Instruments Inc.) with a Co-alloycoated silicon tip (the nominal tip radium is $25 \mathrm{~nm}$ ) using tapping and lift modes. The tip was magnetized along the tip direction. The MFM mapped amplitude/phase shifts in cantilever resonance caused by force gradients. The magnetic properties were measured using a vibrating sample magnetometer in fields up to $15 \mathrm{kOe}$ and also an alternating gradient magnetometer in fields up to $20 \mathrm{kOe}$ at room temperature. The intrinsic magnetic anisotropy $K_{\mathrm{u}}$ was measured using a torque magnetometer in fields up to 15 kOe. Magneto-optical polar Kerr effects were measured using an ultra wide wavelength Kerr spectroscope at photon energy of $1.4-6.8 \mathrm{eV}(182-886 \mathrm{~nm}$ in wavelength). Details of the set-up of the Kerr effect measurement apparatus and the measurements were described in Ref. 8.

\section{Result and Discussion}

\subsection{MFM observations and magnetic properties}

As reported in the previous studies, magnetic stripe and bubble domain patterns were observed for $\mathrm{Co}_{22} \mathrm{Ag} \mathrm{g}_{78}$ granular thin films. ${ }^{73,9)}$ This is similar to that of epitaxial Co films (50-500 $\mathrm{nm}$ thick) which exhibit perpendicular magnetic anisotropy. ${ }^{10}$ ) The presence of the stripe domains indicate the existence of a perpendicular magnetic anisotropy in the $\mathrm{Co}_{22} \mathrm{Ag}_{78}$ granular thin films. Fig. 1 shows a set of MFM images of the DC remenance after parallel magnetization of $15 \mathrm{kOe}$ for the as-deposited sample and the annealed samples in the left column. The corresponding $\mathrm{M}$-H curves with the external field in plane and out-of-plane are also shown in the right column. For comparison, the same Co-alloy-coated silicon tip and the same lift height of $50 \mathrm{~nm}$ were used for the MFM observations. For the as-deposited sample (i.e., $\mathrm{T}_{\mathrm{a}}=300$ $K)$, no discernible stripe domains were observed in the MFM image with respect to the AFM micrograph. With increasing annealing temperature, the contrast of the white and dark magnetic stripes and therefore the magnetic force strength increase from $400 \mathrm{~K}$ to $600 \mathrm{~K}$, while a decrease is observed from $600 \mathrm{~K}$ to $700 \mathrm{~K}$. This indicates a maximum perpendicular magnetic anisotropy at about $600 \mathrm{~K}$. It was also found that the measured width of the aligned magnetic stripes is nearly the same as the as-prepared sample before magnetization, showing a slight increase with the annealing temperature for $300 \mathrm{~K} \leq \mathrm{T}_{\mathrm{a}} \leq 600 \mathrm{~K}$ 
and a slight decrease for $T_{0} \geq 600 \mathrm{~K}$ with a maximum at

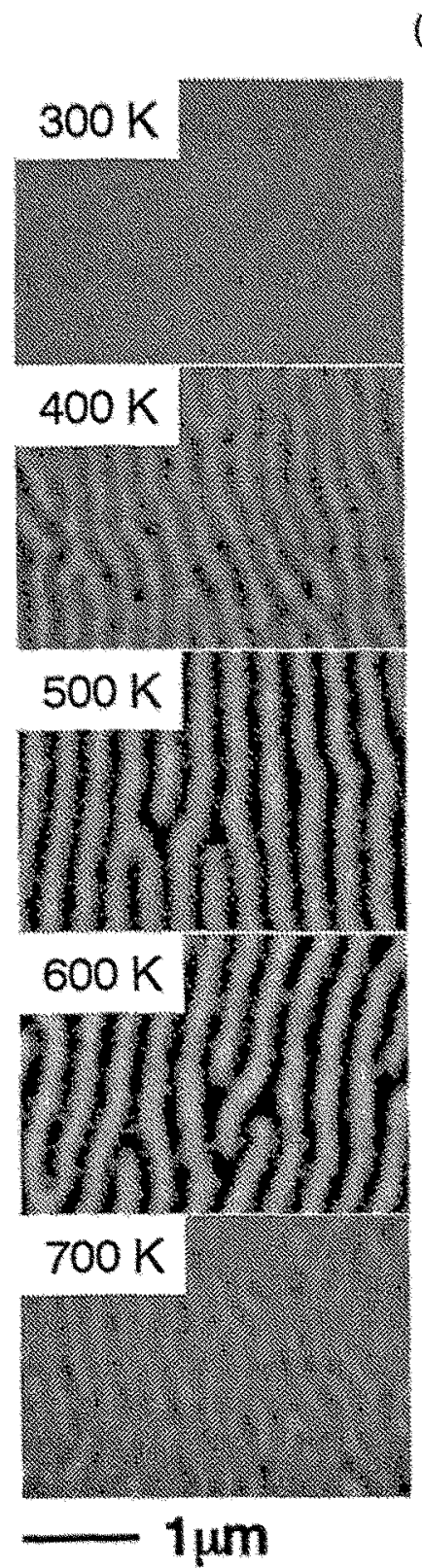

about $600 \mathrm{~K}^{7}$

Fig. 1 MFM images (left) and M-H curves (right) for $\mathrm{Co}_{22} \mathrm{Ag}_{78}$ granular films with different annealing temperatures. MFM observations were performed after a magnetic field $15 \mathrm{kOe}$ was applied in the direction of film plane with the same MFM tip and the same tip-sample separation of $50 \mathrm{~nm}$. The magnetization was normalized with the saturation magnetization. (....... the applied field in the direction of film plane; —- the applied field perpendicular to the film plane.)

From the magnetic hysteresis loops in Hig 1, one can note that for the annealed samples the magnetization is approximately proportional to the applied field up to saturation, and the coercivity and remanence are both close to zero. Due to the linearity, the M-H curves allow us to estimate the magnetic anisotropy energy. Following

Ref. 11, the perpendicular magnetic anisotropy was calculated using the experimental values of parallel and perpendicular saturation fields of the magnetization curves and the saturation magnetization. ${ }^{\text {\% }}$. The calculated perpendicular magnetic anisotropy from the overall magnetization measurements shows a maximum of $5.3 \times$ $10^{5}$ exgs/ce at the anuealing temperature of about $600 \mathrm{~K}$, in agreement with the MFM (of local imaging) results.

A torque magnetometer was used in fields up to 15 kOe to measure the intrinsic magnetic anisotropy $\mathrm{K}_{\mathrm{u}}$, which is determined by the following expression: $\mathrm{K}_{4}=\mathrm{L}_{20}$ $+2 \pi \mathrm{M}_{\mathrm{s}}^{2}$, where $l_{2 \theta}$ is the $2 \theta$ component of the torque amplitude as the maguetic field $\mathrm{H} \rightarrow \infty$. The measured $\mathrm{K}_{\mathrm{u}}$ as a function of $T_{a}$ is shown in Fig. 2. A dotted line is drawn to guide the eyes. It can be seen that the perpendicular magnetic anisotropy shows a maximum of the magnetic anisotropy at about $T_{a}=600 \mathrm{~K}$. This is consistent with MFM observations and magnetization measurements. \%) The saturation magnetization and coercivity vs. annealing temperature are also shown in Fig. 2. It can be seen that both the saturation magnetization and coercivity increase with the annealing temperature due to the increased particle size and the coalescence of the Co particles.

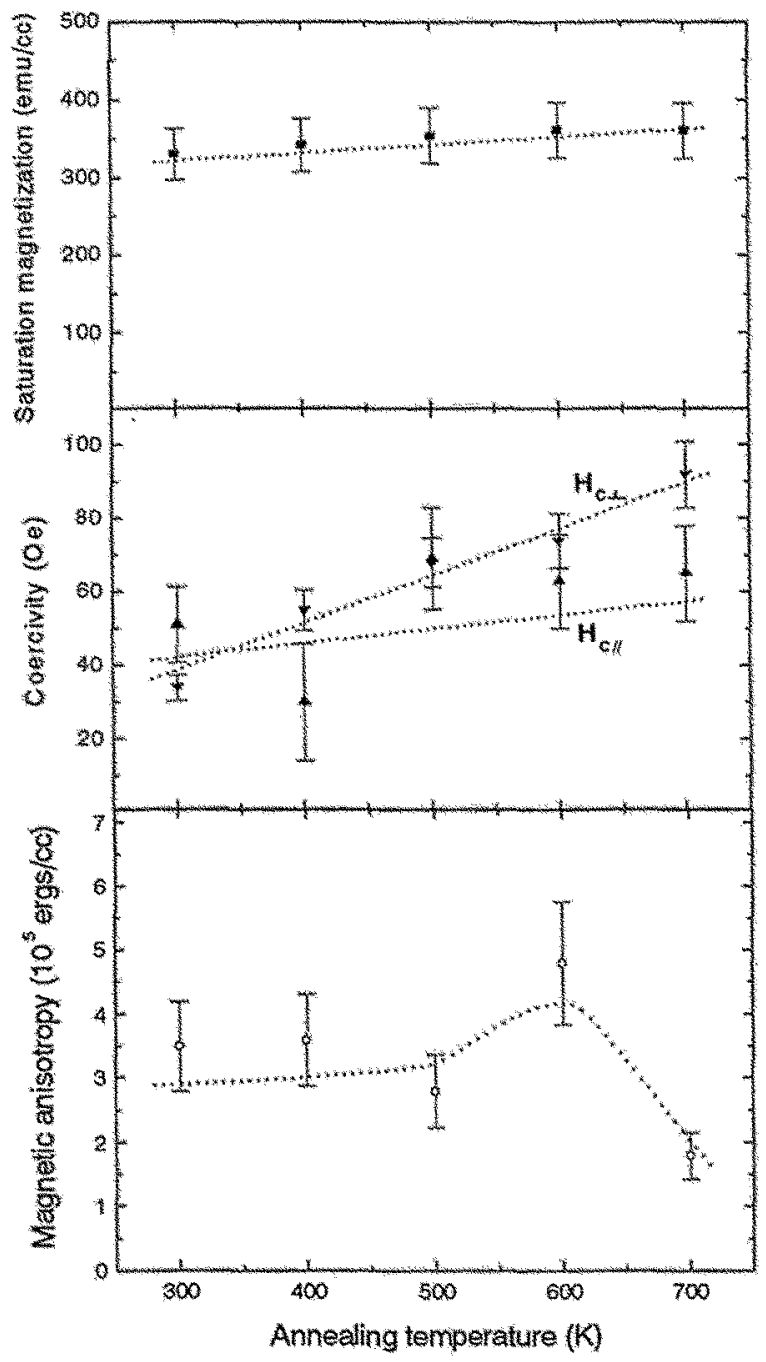


Fig. 2 Measured saturation magnetization, coercivity and intrinsic magnetic anisolropy constant $\mathrm{K}_{\mathrm{p}}$ of $\mathrm{Co}_{22} \mathrm{Ag}_{78}$ granular thin tilms as a function of annealing temperature $\mathrm{T}_{\mathrm{a}+}$

Fig. 3 schematically shows the magnetization distribution in the granular samples. From 3-D view, the stripe/bubble domains are more generally called cylindrical domains. The domains are themselves aligned antiparallel with the nearest-neighbor domains to decrease the net magnetization. It is well known that the cylindrical magnetic domains result from a perpendicular magnetic anisotropy; When the anisotropy energy of a thin film material is higher than the demagnetization energy, the magnetization can be along the easy axis, i.e., surface normal. As observed by MFM, the size of the stripe/bubble domains in $\mathrm{Co}_{22} \mathrm{Ag}_{78}$ is typically of $100 \mathrm{~nm}$ wide, while the single domain Co particles are $\sim 10 \mathrm{~nm}$ in diameter by TEM observations. ${ }^{4)}$ As seen from Fig. 3, the magnetization is not localized at the segregated Co particles, but uniformly distributed. Therefore, Co particles are believed to strongly couple to each other.

The origin of the perpendicular magnetic anisotropy, which leads to the formation of the stripe/bubble domains in the $\mathrm{Co}_{22} \mathrm{Ag}_{78}$ granular films, is not very clear. However, it is proposed that the perpendicular maguetic anisotropy may originate from a $\mathrm{Co}$ Co particle distribution in the direction parallel and perpendicular to the film plane. A further study is needed to clarify this issue. Another possibility is that the perpendicular magnetic anisotropy arises from the surface anisotropy at the interfaces between the Co particles and the Ag matrix.

\subsection{Magneto-optical properties}

Fig. 4 shows the polar Kerr spectrum of the $\mathrm{Co}_{22} \mathrm{Ag}_{78}$ granular films. The spectrum of sputtered FCC Co single film (100 nm thick) is also given as dotted lines in this figure for comparison. ${ }^{8}$ As seen in the figure, the absolute magnitude of Kerr rotation angle $\theta_{\mathrm{K}}$ and Kerr ellipticity $\eta_{K}$ of the $\mathrm{Co}_{22} \mathrm{Ag}_{78}$ granular films is smaller than that of the Co single film over the whole measured photon energy. The spectral feature is similar to the Co spectra. The $\theta_{\mathrm{K}}$ exhibits a positive peak at $5-6 \mathrm{eV}$, and the $\eta_{\mathrm{K}}$ has a negative peak at $4-5 \mathrm{eV}$. However, both peak positions shift toward lower energies compared with the Kerr spectra of the sputtered pure Co film. As seen, the Kerr effects are enhanced with annealing (the amplitudes of both peaks increase with the annealing temperature). Furthermore, both peak positions decrease with increasing annealing temperature (for the as-deposited sample, both peaks are very broad compared with the annealed samples) as shown in Fig. 5. Also shown in Fig. 5 is the average Co particle size as a function of annealing temperature. One can note that the average Co particle size increases with the annealing temperature. ${ }^{4}$ This indicates that the enhancement of Kerr effects and the peak shifts must be attributed to the change in electronic structures of Co granules / the change of the shape of the particles. ${ }^{4)}$

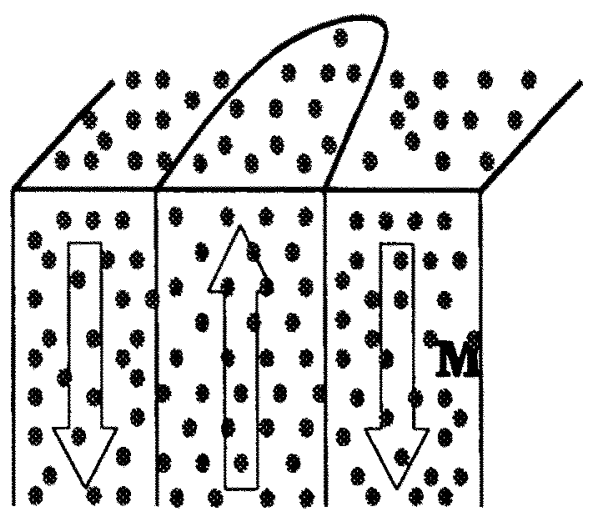

Fig. 3 Schematic of magnetization distribution in $\mathrm{Co}_{22} \mathrm{Ag}_{78}$ granular films. (Solid circle: Co particle)

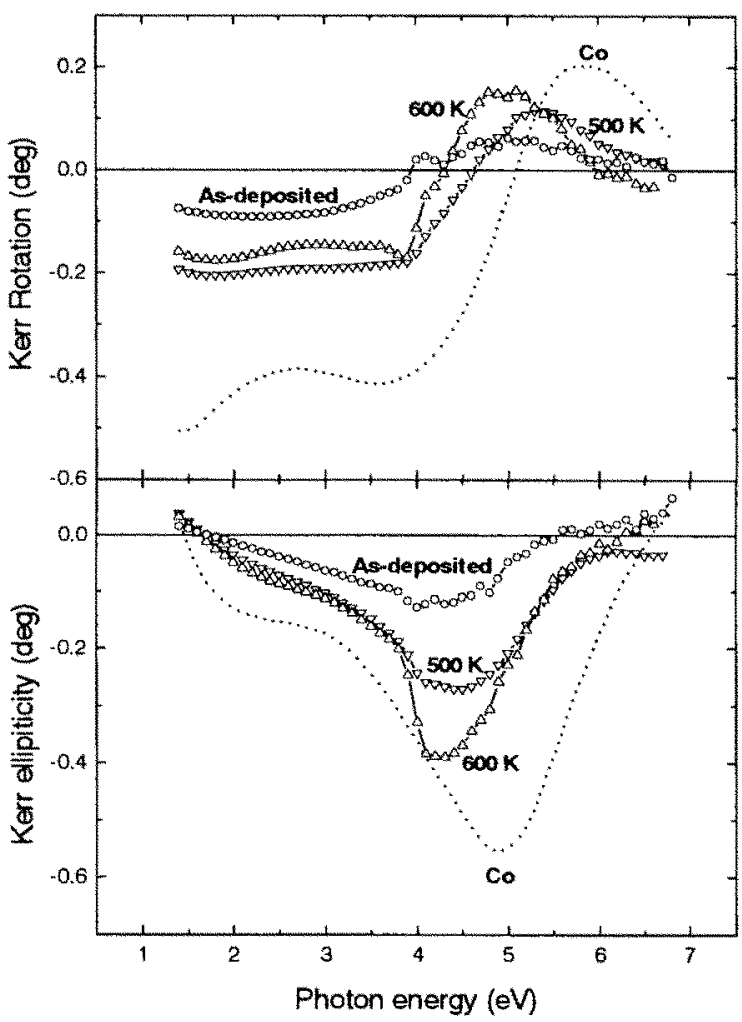

Fig. 4 Kerr rotation angle $\theta_{K}$ and Kerr ellipticity $\eta_{K}$ of $\mathrm{Co}_{22} \mathrm{Ag}_{78}$ granular films as a function of photon energy. The spectra of sputtered FCC Co film are also shown in this figure as dotted lines for comparison. 


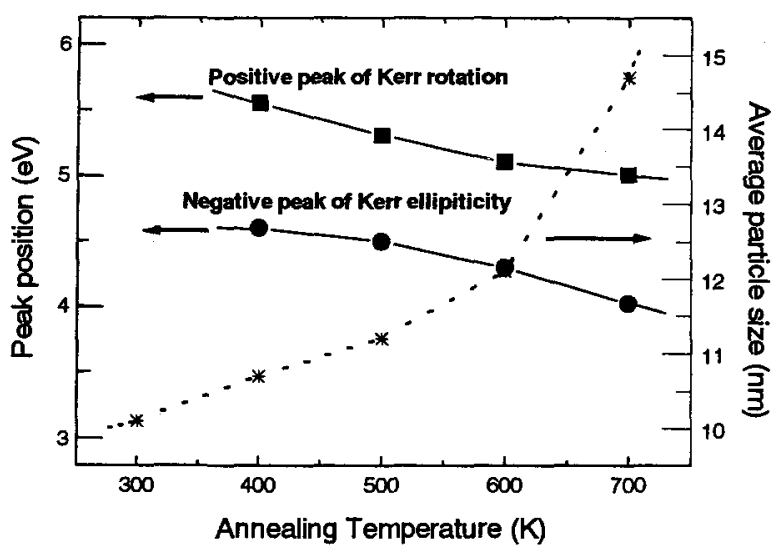

Fig. 5 Peak positions of the positive peak at $5-6 \mathrm{eV}$ of Kerr rotation angle and the negative peak at $4-5 \mathrm{eV}$ of Kerr ellipiticity in $\mathrm{Co}_{22} \mathrm{Ag}_{78}$ granular thin films vs. annealing temperature. Also shown in this figure is the average Co particle diameter measured by TEM observations.

\section{Conclusion}

In summary, the magnetic domain patterns and the perpendicular magnetic anisotropy have been investigated by MFM, magnetization and torque measurements. The appearance and characteristics of both the stripe and bubble domains observed by MFM strongly suggest the existence of a perpendicular magnetic anisotropy. The measured values of the perpendicular magnetic anisotropy by magnetization and torque measurements show a maximum of about $5 \times 10^{5} \mathrm{ergs} / \mathrm{cc}$ at the annealing temperature of about $600 \mathrm{~K}$. This perpendicular anisotropy may originate from an anisotropic $\mathrm{Co}-\mathrm{Co}$ particle distribution due to the co-deposition of $\mathrm{Co}$ and $\mathrm{Ag}$ and surface anisotropy at the interfaces between the Co granules and the $\mathrm{Ag}$ matrix. Finally, the magneto-optical
Kerr effect in $\mathrm{Co}_{22} \mathrm{Ag}_{78}$ granular thin films is measured using a Kerr spectroscope at photon energy of 1.4-6.8 eV. The observed shift of both the positive peak at 5-6 eV of Kerr rotation angle and the negative peak at $4-5 \mathrm{eV}$ of the Kerr ellipticity toward lower energies with the increasing annealing temperature is believed to be attributed to the change in electronic structures of Co particles / the change of the shape of the particles.

The present work was partly supported by The Sumitomo Foundation (1997 1998) and The Grant-in-Aid for International Scientific Research (Joint Research \#09044186) supported by The Ministry of Education, Science, Sports and Culture.

\section{References}

1) E. A. Gan'shina, A. B. Granovsky, V. S. Guschin, N. S. Perov, and A. A. Radkovskaya, J. Magn. Magn. Mater. 160, 335 (1996).

2) T. Katayama, et. al., J. Magn. Soc. Japan. Suppl. 19 (S1), 243 (1995).

3) H. Sang, Z. S. Jiang, G. Guo, J. T. Li, S. Y. Zhang, and Y. W. Du, J. Magn. Magn. Mater. 140-144, 589 (1995).

4) H. Sang, S. Y. Zhang, H. Chen, G. Ni, J. M. Hong, X. N. Zhao, Z. S. Jiang, and Y. W. Du, Appl. Phys. Lett. 67, 2017 (1995).

5) H. Sang, N. Xu, J. H. Du, G. Ni, S. Y. Zhang, and Y. W. Du, Phys. Rev. B 53, 15023 (1996).

6) H. Sang, G. Ni, J. Lu, H. Chen, J. R. Zhang, and Y. W. Du, J. Vac. Sci. Technol. B 15, 1456 (1997).

7) Y. J. Chen, W. Y. Cheung, I. H. Wilson, N. Ke, S. P. Wong, J. B. Xu, H. Sang and G. Ni, Appl. Phys. Lett. 72, 2472 (1998).

8) W. P. Van Drent and T. Suzuki, J. Magn. Magn. Mater. 175, 53 (1997).

9) Y. J. Chen, T. Suzuki, S. P. Wong, and H. Sang, presented at MMM'98, Miami, Nov. 8-12, 1998.

10) M. Hehn, S. Padovani, K. Ounadjela, and J. P. Bucher, Phys. Rev. B 54, 3428 (1996).

11) J. Q. Xiao, C. L. Chien, and A. Gavrin, J. Appl. Phys. 79, 5309 (1996). 